\title{
La intervención de terceros Estados ante la Corte Internacional de Justicia: identificando criterios jurisprudenciales para la aceptación o denegación de las solicitudes
}

\author{
Josselyn Roca Calderón*
}

\section{RESUMEN}

Existen tres supuestos posibles de intervención de terceros Estados en las controversias ante la Corte Internacional de Justicia. La intervención por interés de orden jurídico y la intervención por la interpretación de una convención se encuentran estipuladas en el Estatuto de la CIJ, pero la intervención por necesidad de consentimiento de una parte indispensable ha sido establecida en la jurisprudencia del referido tribunal. Dentro de este artículo se detallará cada supuesto, así como los lineamientos y/o criterios establecidos por la propia CIJ, los cuales han dotado de contenido a los conceptos establecidos en sus disposiciones, ya sea en el Estatuto o en su Reglamento. Para estos efectos, se analizará su jurisprudencia en casos con solicitudes de intervención a fin de identificar denominadores comunes en sus pronunciamientos.

Palabras clave: Corte Internacional de Justicia, Estatuto, interés de orden jurídico, interpretación de una convención, intervención de terceros Estados, jurisprudencia, parte indispensable.

\footnotetext{
Abogada con segunda especialidad en Derecho Internacional Público por la Pontificia Universidad Católica del Perú, presidenta de la Asociación Civil Ius Inter Gentes de la PUCP e investigadora del Proyecto 70817 sobre respuestas del derecho internacional a la corrupción asociada al crimen transnacional adjudicado por el Ministerio de Ciencias de Colombia y por la Universidad del Rosario de Colombia. Correo electrónico: jrocac@pucp.pe

iD https://orcid.org/0000-0002-8776-4593
} 
Intervention by third States before the International Court of Justice: Identifying case law criteria for the acceptance or denial of requests

\section{Abstract}

There are three possible types of intervention by third States in the disputes before the International Court of Justice. Intervention for the interest of a legal nature and intervention for the construction of a convention are stipulated in the ICJ Statute, but intervention by necessity of consent of an indispensable party has been established in the jurisprudence of the aforementioned court. Within this article each type will be detailed, as well as the guidelines and/or criteria established by the ICJ itself, which have provided content to the concepts established in its provisions, either in the Statute or in its Regulations. For these purposes, their jurisprudence will be analyzed in cases with requests for intervention to identify common denominators in their pronouncements.

Keywords: International Court of Justice, Statute, interest of a legal nature, construction of a convention, intervention of third States, jurisprudence, indispensable party.

\section{Introducción}

El presente artículo versará sobre el estudio de los diferentes tipos de intervención de terceros Estados que pueden suscitarse en el marco de una controversia internacional ante la Corte Internacional de Justicia (en adelante, «la CIJ» o «la Corte»). Estos tipos de intervención cuentan con sus propios criterios y requisitos establecidos en el Estatuto y el Reglamento de la CIJ. Sin embargo, al no ser estos totalmente específicos, el referido tribunal, a través de su jurisprudencia, ha estipulado lineamientos que deberán ser considerados al momento de requerir la intervención en un litigio ante él. Cabe precisar que el supuesto de parte indispensable no se encuentra establecido por las fuentes antes señaladas, ya que su origen radica en la jurisprudencia de la Corte.

Los criterios, así como los requisitos, serán mencionados con ayuda de la doctrina y la jurisprudencia de la CIJ, así como su Estatuto y Reglamento. Asimismo, luego de explicar en qué consiste cada supuesto, procederemos a brindar los casos necesarios para un mayor entendimiento de los tipos de intervenciones, en los cuales observaremos cómo algunos fueron admitidos acorde a derecho, y cómo, en otros, quizá la CIJ estableció un estándar un poco elevado, así como también casos en los que se deslizaba la hipótesis de que era posible solicitar la intervención.

Por último, en el transcurso del análisis, apreciaremos ciertos patrones que nos describen la forma de actuar de la CIJ. Es así como verificaremos que, por ejemplo, solo en cinco casos de diecisiete se ha admitido la solicitud de intervención, denegando, así, al resto bajo criterios particulares, en los que prevalecerían las consideraciones de las partes en controversia. Cabe precisar que solo en una solicitud de 
intervención, la CIJ decidió ir en contra de la objeción presentada por parte de los Estados en litigio ante el requerimiento de participar en el proceso. En ese sentido, reflexionaremos sobre el actuar de la $\mathrm{CIJ}$, el cual no ha seguido un mismo criterio uniforme.

\section{Los diferentes tipos de intervención de terceros Estados ante la Corte Internacional de Justicia}

Las controversias internacionales, entendidas como «[...] un desacuerdo o discrepancia, sobre alguna materia jurídica o una situación de hecho, cuya existencia no depende del reconocimiento de las partes, pues tiene una existencia propia y objetiva» (Novak y García-Corrochano, 2019, p. 449), sometidas ante la CIJ, son comúnmente bilaterales. Sin embargo, es jurídicamente posible que algún tercer Estado intervenga en la controversia, en razón de que 1) los efectos del fallo puedan impactar directamente en este, 2) para resolver la controversia se requiera de una interpretación idónea de un tratado en el cual sea parte y/o 3) el caso requiera necesariamente la intervención de este para poder ser resuelta por la CIJ.

Las denominaciones formales de los referidos supuestos de intervención, establecidos en el Estatuto y en la jurisprudencia de la CIJ, son los siguientes: por la existencia de un interés de orden jurídico (artículo 62 del Estatuto de la CIJ), por la interpretación de una convención (artículo 63 del Estatuto de la CIJ) y por ser parte indispensable (jurisprudencia de la CIJ). Cada uno de estos será definido conforme a los establecido en los instrumentos jurídicos de la CIJ, teniendo en cuanta, además, la jurisprudencia propia de la Corte en los diversos casos de intervención de terceros Estados, los cuales serán apreciados en el desarrollo del presente apartado. Cabe precisar que el caso Monetary Gold Removed from Rome in 1943 no es contabilizado en los casos en que terceros Estados solicitaron intervenir, ya que la CIJ fue la que solicitó la intervención, pues sin la intervención del tercero, no le era posible emitir pronunciamiento.

Por último, ante un primer acercamiento de los conceptos de los diferentes supuestos mencionados, se podría considerar que estos responden a la necesidad de resolver la controversia lo más acorde a derecho o, también, a fin de proteger a terceros Estados que puedan verse afectados por el resultado del fallo de la CIJ; sin embargo, del estudio, podríamos adelantarnos en afirmar que el tribunal considera a la intervención del tercer Estado más como «una intrusión que como un medio para asegurar una mejor disputa» (Bonafé, 2018, p. 98). Este adelanto de opinión respondería a los patrones registrados y a la renuencia de la admisión de las solicitudes de intervención formuladas por terceros Estados. 


\subsection{Intervención de terceros Estados por la existencia de un interés de orden jurídico}

Consideramos importante señalar que este supuesto de intervención de terceros Estados por la existencia del interés de orden jurídico, estipulado en el artículo 62 del Estatuto de la CIJ, no es originario de la CIJ, sino que data de la Corte Permanente de Justicia Internacional, ante la cual solo se presentó una solicitud de intervención de este tipo en el caso S.S Wimbledon (Solimano, 2013, p. 24). Por esto último, el desarrollo del referido supuesto se ha suscitado más bien en el marco de la CIJ. Asimismo, debido a la poca admisión de dicho supuesto ante la CIJ, se podría afirmar que no existe mucho desarrollo sobre esta materia.

El referido tipo de intervención consiste en que un Estado alegue la existencia de un interés de orden jurídico que puede verse afectado por la decisión de la CIJ sobre el litigio. Este supuesto requiere de tres requisitos básicamente, conforme a lo reflejado en el artículo 62 del Estatuto de la CIJ y añadido por la jurisprudencia del referido tribunal: 1) un interés de orden jurídico, 2) el objeto de la intervención y 3) el vínculo jurisdiccional, es decir, el fundamento de la competencia, todo lo cual debe constar en la demanda de intervención (Novak y García-Corrochano, 2019, p. 600). Estos requisitos han constituido puntos controvertidos para la CIJ en diversas oportunidades. Cabe precisar que es sobre el primer y el tercer requisito que existe mayor desacuerdo entre los publicistas, ya que suelen considerar que solo existirá interés jurídico cuando haya un vínculo jurisdiccional demostrado, siendo, por ejemplo, para Cahier, innecesario indicar la base de la competencia (citado por Novak y García-Corrochano, 2019, p. 601).

Como podemos observar, este tipo de intervención responde a que haya una posible afectación, lo cual, en gran medida, es complejo de demostrar, pero lo más controversial es que la disposición no define qué se deberá entender por «orden jurídico». Sobre esta terminología, Bonafé señala que no implica la posible afectación de un derecho del Estado, sino simplemente de un interés, pero dicho Estado «deberá demostrar que el interés es objeto de una reivindicación real y concreta, basado en el derecho internacional y que debe estar conectado con el tema de la controversia presentada ante la Corte» (2018, p. 99). Dicho requerimiento, conforme a la autora, resulta cuestionable y, a veces, poco probable de sostener ante la solicitud dirigida a la CIJ, ya que algunas veces puede tratarse de la afectación de un derecho, en vez de un mero interés.

Respecto a lo dicho, vamos a observar que la CIJ no es uniforme al aplicar los criterios brevemente señalados en sus propios instrumentos; así también, apreciaremos que evita admitir solicitudes de intervención si las partes en controversia así lo desean 
y que prefiere, más bien, ser cuidadosa en su fallo de tal manera que no afecte a un tercer Estado. Asimismo, se podrá observar que sus estándares para admitir algún petitorio de aquel tipo son bastantes altos y a veces cuestionables, ya que, en algunos casos, califica que la solicitud es muy general y, en otras, señala que es muy específica.

Sobre esto, un ejemplo vendría a suscitarse en el caso denominado Continental Shelf (Tunisia/Libyan Arab Jamahiriya), en el cual el Estado de Malta solicitó intervenir conforme el artículo 62 del Estatuto de la CIJ. La razón por la que Malta había requerido intervenir, versaba debido a que consideraba que el fallo respecto a la delimitación de la plataforma continental de los Estados en controversias podría ser perjudicial, toda vez que Malta tenía sus propias disputas al respecto con ambos Estados. En este caso, el interés existente fue reconocido por la CIJ, toda vez que, en su sentencia sobre la solicitud de intervención, manifestó:

$[\ldots]$ it has a certain interest in the Court's treatment of the physical factors and legal considerations relevant to the delimitation of the continental shelf boundaries of States within the central Mediterranean region that is somewhat more specific and direct than that of States outside that region. (1981b, párrafo 33)

Sin embargo, para la Corte el interés que argumentaba Malta era uno común a otros Estados de la región, por lo que consideraba que lo requerido y señalado por dicho Estado era muy general e insuficiente para circunscribirse dentro del supuesto del artículo 62 del Estatuto. Asimismo, dentro de su sentencia añadió que lo que Malta debería evidenciar era "an interest of a legal nature which may be affected by the Court's decision» (CIJ, 1981b, párrafo 33). En ese sentido, podemos apreciar en el caso que el referido tribunal, explícitamente, manifestó que el interés señalado en el artículo 62 no requiere que sea simple y general, sino que tenga una naturaleza jurídica. Sin embargo, continuó en no dotar de contenido a este requerimiento.

Asimismo, en el referido caso, Libia y la propia Corte refieren a la importancia del vínculo jurisdiccional entre las partes y el Estado que desea intervenir como base para evidenciar el interés. Sin embargo, es otro criterio que la propia CIJ no desarrolla e incluso menciona que, en el caso de ensayos nucleares, se cuestionó si efectivamente era necesario el referido vínculo para que un tercer Estado pueda intervenir (CIJ, 1981b, párrafo 32). Cabe precisar que la solicitud que hemos estado comentando fue rechazada por la CIJ por unanimidad (CIJ, 1981b, párrafo 37).

Por otro lado, apreciaremos el caso de intervención denominado Continental Shelf (Libyan Arab Jamahiriya/Malta), de 1984, en el que Italia presentó su solicitud, pero dicho Estado, a fin de evitar que su requerimiento sea rechazado, fue bastante preciso en cuanto al interés alegado. De hecho, «incluso estipuló las coordenadas de los puntos en los cuales se encontrarían las fronteras marítimas entre los tres países» 
(Solimano, 2013, p. 27), ya que poseía la preocupación de que el fallo de la CIJ afectara su soberanía en cuanto a la zona marítima en disputa. No obstante, a pesar de la diligencia de dicho Estado, la CIJ consideró que Italia estaba introduciendo una nueva controversia al caso ya preexistente, por lo que, declaró improcedente el requerimiento (CIJ, 1984, párrafo 34).

De lo expuesto, se puede apreciar que la CIJ se ha pronunciado de manera distinta sobre los criterios exigibles ante la solicitud de intervención de terceros Estados. En el caso presentado sobre el requerimiento de Malta, podemos apreciar que, para la CIJ, el Estado en mención no poseía un interés específico, sino que podía ser similar al de otros Estados de la región, por lo que lo consideraba muy general. Asimismo, no aprovechó para manifestar qué se debe entender por interés de naturaleza jurídica, y tampoco dotó de contenido al vínculo jurisdiccional que mencionó en su fallo. En contraposición, la CIJ rechazó la solicitud de intervención de Italia, la cual precisó la afectación en específico, al entender que, al haber sido tan específico el requerimiento, estaría buscando introducir una nueva controversia.

Por otra parte, cabe precisar que, en ambos casos, las partes en litigio no estaban de acuerdo con la intervención de un tercer Estado, quienes tuvieron la oportunidad de pronunciar sus motivos por los cuales no consideraban que tanto Malta como Italia debieran intervenir (CIJ, 1981a, pp. 267, 275; 1983, pp. 437, 448). Sin embargo, en el caso Land and Maritime Boundary between Cameroon and Nigeria (Cameroon v. Nigeria: Equatorial Guinea intervening), Camerún y Nigeria no se opusieron a la intervención de Guinea Ecuatorial y, por tanto, la CIJ decidió aceptar el requerimiento. Incluso, resaltó como un considerando en su providencia que ninguna de las partes objetó lo solicitado por el tercer Estado (CIJ, 1999b, párrafo 12). En ese sentido, la manifestación de voluntad de los Estados para que un tercero pueda intervenir parecería ser un criterio subjetivo que la CIJ ha adoptado en su jurisprudencia.

Asimismo, los tres casos mencionados han llevado a considerar que no bastará que el tercer Estado alegue simples intereses, sino que será relevante que precise el derecho posible de ser afectado. En el último caso citado, apreciamos que el Estado solicitante señaló lo siguiente: «Equatorial Guinea states that the purpose of its intervention is "to protect [its] legal rights in the Gulf of Guinea by all legal means"” (CIJ, 1999a, p.1). $\mathrm{Al}$ respecto, el referido tribunal señaló que, efectivamente, la controversia de delimitación entre Camerún y Nigeria podría conllevar a la vulneración de un derecho, confirmando así lo alegado por Guinea, respecto a que los Estados en controversia estarían ignorando una línea media que lo involucraría, por lo que, un resultado sin considerar su participación podría haber resultado en la explotación de recursos que le pertenecerían. 
Cabe precisar que, conforme a lo señalado por Solimano, solamente en el caso Jurisdictional Immunities of the State (Germany v. Italy: Greece intervening), al parecer, se habría alegado un simple interés que habría sido aceptado por la CIJ, pero en la opinión disidente, el juez Gaja «llamó la atención al respecto, al afirmar que el interés reclamado por Grecia no tenía sustento en el derecho internacional» (Solimano, 2013, p. 56). Esta opinión del referido magistrado pareciese que se condice a lo que reflejan los fallos anteriores de la CIJ respecto de la figura estudiada, ya que se prefería la demostración por parte del Estado de la afectación a un derecho que a un simple interés. Así, el interés que alegaba Grecia era solamente «to inform the Court of the nature of the legal rights and interests of Greece that could be affected by the Court's decision in light of the claims advanced by Germany to the case before the Court》 (CIJ, 2011, párrafo 28) y, en dicha línea, finalmente la Corte solo autorizó la intervención limitada al tema de las sentencias de cortes griegas declaradas por las italianas como ejecutables en Italia (Negro, 2012, p. 247). No obstante, la CIJ también resalta el hecho de que las partes no se opusieron a la solicitud (CIJ, 2011, párrafo 5).

Por lo expuesto, podemos identificar que la renuencia de la CIJ en aceptar la intervención de terceros Estados puede deberse a diversas razones:

- la consideración general de que el fallo no afecta los derechos de los terceros, la misma que, de hecho, se encuentra reflejada en el artículo 59 del Estatuto de la CIJ, según el cual la sentencia de la CIJ no es obligatoria sino a las partes;

- la exigibilidad de una posible afectación de derecho (aunque también quepa extraordinariamente la posibilidad de que se acepte en cuanto a meros intereses) como requisito para permitir la intervención; y,

- la precaución de la CIJ en que la solicitud no la direccione a analizar sobre una controversia no alegada por las partes en litigio y, por tanto, seguir brindándoles seguridad y, con mayor razón, cuando objetan la solicitud.

Por último, consideramos pertinente mencionar los casos en los que se solicitó la intervención de un tercer estado en virtud del artículo 62 del Estatuto de la CIJ a través del presente cuadro ${ }^{1}$ :

\footnotetext{
1 Elaboración propia a partir de la información presente en la página oficial de la CIJ, los casos se encuentran ordenados cronológicamente del más antiguo al más reciente.
} 


\begin{tabular}{|c|c|c|c|c|}
\hline Caso (nombre oficial en inglés) & $\begin{array}{c}\text { Estado } \\
\text { solicitante }\end{array}$ & Artículo & $\begin{array}{l}\text { Autori- } \\
\text { zación }\end{array}$ & Instrumento \\
\hline Nuclear Tests (Australia v. France) & \multirow[b]{2}{*}{ Fiji } & \multirow[b]{2}{*}{62} & \multirow[b]{2}{*}{ No } & \multirow[b]{2}{*}{ Providencia } \\
\hline $\begin{array}{l}\text { Nuclear Tests (New Zealand v. } \\
\text { France) }\end{array}$ & & & & \\
\hline $\begin{array}{l}\text { Continental Shelf (Tunisia/Libyan } \\
\text { Arab Jamahiriya) }\end{array}$ & Malta & 62 & No & Sentencia \\
\hline $\begin{array}{l}\text { Continental Shelf (Libyan Arab } \\
\text { Jamahiriya/Malta) }\end{array}$ & Italia & 62 & No & Sentencia \\
\hline $\begin{array}{l}\text { Land, Island and Maritime Frontier } \\
\text { Dispute (El Salvador/Honduras: } \\
\text { Nicaragua intervening) }\end{array}$ & Nicaragua & 62 & Sí & $\begin{array}{c}\text { Providencia y } \\
\text { sentencia }\end{array}$ \\
\hline $\begin{array}{l}\text { Land and Maritime Boundary } \\
\text { between Cameroon and Nigeria } \\
\text { (Cameroon v. Nigeria: Equatorial } \\
\text { Guinea intervening) }\end{array}$ & $\begin{array}{l}\text { Guinea } \\
\text { Ecuatorial }\end{array}$ & 62 & Sí & Providencia \\
\hline \multirow{5}{*}{$\begin{array}{l}\text { Request for an Examination of } \\
\text { the Situation in Accordance with } \\
\text { Paragraph } 63 \text { of the Court's Judgment } \\
\text { of } 20 \text { December } 1974 \text { in the Nuclear } \\
\text { Tests (New Zealand v. France) Case }\end{array}$} & Australia & 62 & \multirow{5}{*}{ No } & \multirow{5}{*}{ Providencia } \\
\hline & Islas Salomón & \multirow{4}{*}{62 y 63} & & \\
\hline & Islas Marshall & & & \\
\hline & $\begin{array}{l}\text { Estados } \\
\text { Federados de } \\
\text { Micronesia }\end{array}$ & & & \\
\hline & Islas Samoa & & & \\
\hline $\begin{array}{l}\text { Sovereignty over Pulau Ligitan and } \\
\text { Pulau Sipadan (Indonesia/Malaysia) }\end{array}$ & Filipinas & 62 & No & Sentencia \\
\hline \multirow{2}{*}{$\begin{array}{l}\text { Territorial and Maritime Dispute } \\
\text { (Nicaragua v. Colombia) }\end{array}$} & Costa Rica & \multirow{2}{*}{62} & \multirow{2}{*}{ No } & Sentencia \\
\hline & Honduras & & & Sentencia \\
\hline $\begin{array}{l}\text { Jurisdictional Immunities of the State } \\
\text { (Germany } v \text {. Italy: Greece intervening) }\end{array}$ & Grecia & 62 & Sí & Providencia \\
\hline
\end{tabular}


Del cuadro expuesto, podemos observar que, como ya habíamos estado detallando, la CIJ, por el supuesto del artículo 62 de su Estatuto, solo autorizó la solicitud de un tercer Estado en tres casos. Asimismo, vamos a apreciar que cabe la posibilidad de alegar los dos tipos de intervención que están estipulados en el Estatuto al mismo tiempo, lo cual se visualiza en el caso denominado Request for an Examination of the Situation in Accordance with Paragraph 63 of the Court's Judgment of 20 December 1974 in the Nuclear Tests (New Zealand v. France), en el cual distintos Estados insulares solicitaron intervenir en virtud de ambas disposiciones.

\subsection{Intervención de terceros Estados por la interpretación de una convención}

Este tipo de intervención, estipulado en el artículo 63 del Estatuto de la CIJ, se refiere al derecho que tendrán terceros Estados de participar de la controversia, siempre que la misma suponga la interpretación de un tratado en la que sean partes, al igual que los Estados en litigio. Al respecto, el punto controversial de este supuesto radica en que, al parecer, debería estar contenido también en el artículo 62 del Estatuto, ya que, como bien recordamos, este último artículo menciona que un Estado podrá intervenir cuando exista un interés de orden jurídico. Consideramos que no existe impedimento alguno para afirmar que la interpretación de un tratado para un tercer Estado pueda ser un tipo de interés jurídico. Es por ello que consideramos que el tipo de intervención al cual nos referimos en este apartado (artículo 63) es una especie del género que es la intervención por interés de orden jurídico (artículo 62).

De igual forma, entendemos que lo referido al artículo 62, al tratarse de distintos supuestos de interés de orden jurídico, deberá estar sujeto a la evaluación y decisión de la CIJ respecto a la admisión. No obstante, en el caso del supuesto del artículo 63, al tratarse de los efectos sustanciales que implica ser parte de un tratado y respetar lo pactado por las partes en concordancia con la soberanía de cada Estado, es que dicha disposición es observada como un derecho que tienen los Estados de intervenir. Por ello queda a la libre discreción de cada Estado el participar o no ante la solicitud de la CIJ de ser tercero interviniente, lo cual no sucede en el supuesto del artículo 62, ya que, en dicho supuesto, la Corte cuenta con una mayor discrecionalidad (CIJ, 2011, párrafo 32). Al respecto, cabe precisar que, de aceptar los terceros Estados intervenir, una vez notificados, el fallo será igualmente obligatorio para aquellos terceros Estados en lo relativo a la interpretación del tratado por parte de la CIJ.

Este supuesto parece ser menos controversial que el establecido en el artículo 62, ya que se presupone que solo quedaría a discreción del tercer estado interesado en intervenir; sin embargo, vamos a observar que aquello no se ajusta completamente a la práctica, ya que, si ese fuese el caso, la CIJ no tendría la facultad de rechazar solicitudes de intervenir en cuanto refiera a la interpretación de tratados, como lo hizo 
en el caso Military and Paramilitary Activities in and against Nicaragua (Nicaragua v. United States of America), en el que El Salvador requirió intervenir, pero su solicitud no fue admitida.

Esto se debió a que, si bien El Salvador sí era parte de la Carta de las Naciones Unidas, al igual que las partes en litigio, la CIJ misma había establecido, en una providencia anterior, que el procedimiento escrito se dirigiría inicialmente de manera exclusiva a las cuestiones de admisibilidad y competencia, por lo que la solicitud remitida por El Salvador dos días antes de cerrar ese procedimiento fue declarada inadmisible (CIJ, 1986, párrafo 7). Es importante señalar que esto se dio sin perjuicio de sí haberse cumplido con la etapa procedimental establecida en el artículo 82 del Reglamento de la CIJ, que únicamente exige depositar una declaración antes del procedimiento oral. En ese sentido, si bien la CIJ no cuenta con el mismo nivel de discrecionalidad que en el supuesto del artículo 62 de su Estatuto, sí cuenta, en la práctica, con la posibilidad de fijar plazos más estrictos que los establecidos en su Reglamento para efectos de aceptar o no las solicitudes de intervención en virtud de este supuesto.

Por otro lado, nos parece importante dejar de manifiesto la interrogante del por qué en el artículo 63 del Estatuto de la CIJ no se han incluido a otras fuentes formales del derecho internacional como la costumbre internacional o los principios generales del derecho internacional, entre otras, más allá de la interpretación de un tratado. La incógnita responde a que, si en cuanto a tratados se busca la interpretación de las normas jurídicas a las cuales se han visto obligadas las partes, no comprenderíamos por qué no se podría buscar lo propio en cuanto a los cuestionamientos que surjan de una costumbre internacional, más aún si se suscitan sobre costumbres regionales. Consideramos que el referido artículo debería abarcar la interpretación de todo tipo de fuentes de derecho internacional que generan posibles obligaciones jurídicas entre las partes.

Por otro lado, observamos que existen menos solicitudes realizadas a intervenir en cuanto a la interpretación de un tratado. A nuestro entender, esto respondería a que el fallo de la CIJ, respecto al entendimiento del tratado, vendría a ser vinculante para el tercer Estado solicitante aceptante, ya que así lo establece expresamente el artículo 63 del Estatuto, lo cual es algo a lo que no siempre querrán someterse los Estados por la posibilidad de que el resultado les sea perjudicial. Asimismo, cabe señalar que, si bien es cierto que, por lo general, la CIJ se pronuncia de tal manera que no afecte a terceros Estados, en este caso queda descartado totalmente que el fallo del referido tribunal tenga implicancias jurídicas para el tercero que decidió no intervenir, ya que, como se dijo, se trata de un derecho del Estado, y la CIJ no podría interpretar un tratado contrariamente a la voluntad de sus partes en caso de que estas no formen parte de la controversia. 
Por último, consideramos pertinente mencionar las solicitudes realizadas en cuanto a la intervención de terceros estados conforme al artículo 63 del Estatuto de la CIJ, así como detallar en qué casos dicho ente se pronunció a favor y en qué casos manifestó su negativa a través del presente cuadro ${ }^{2:}$

\begin{tabular}{|c|c|c|c|c|}
\hline Caso (nombre oficial en inglés) & $\begin{array}{l}\text { Estados } \\
\text { solicitantes }\end{array}$ & Artículo & $\begin{array}{l}\text { Autori- } \\
\text { zación }\end{array}$ & Instrumento \\
\hline Haya de la Torre (Colombia v. Peru) & Cuba & 63 & Sí & $\begin{array}{l}\text { Sentencia (de } \\
\text { fondo) }\end{array}$ \\
\hline $\begin{array}{l}\text { Military and Paramilitary } \\
\text { Activities in and against Nicaragua } \\
\text { (Nicaragua } v \text {. United States of } \\
\text { America) }\end{array}$ & El Salvador & 63 & No & Providencia \\
\hline \multirow{4}{*}{$\begin{array}{l}\text { Request for an Examination } \\
\text { of the Situation in Accordance } \\
\text { with Paragraph } 63 \text { of the Court's } \\
\text { Judgment of } 20 \text { December } 1974 \text { in } \\
\text { the Nuclear Tests (New Zealand } v \text {. } \\
\text { France) Case }\end{array}$} & Islas Salomón & \multirow{4}{*}{62 y 63} & \multirow{4}{*}{ No } & \multirow{4}{*}{ Providencia } \\
\hline & Islas Marshall & & & \\
\hline & $\begin{array}{c}\text { Estados Federados } \\
\text { de Micronesia }\end{array}$ & & & \\
\hline & Islas Samoa & & & \\
\hline $\begin{array}{l}\text { Whaling in the Antarctic (Australia } \\
\text { v. Japan: New Zealand intervening) }\end{array}$ & Nueva Zelanda & 63 & Sí & Providencia \\
\hline
\end{tabular}

\subsection{Intervención de un tercer Estado por ser parte indispensable}

Este supuesto no se encuentra establecido en el Estatuto ni en ninguna disposición en el marco de la CIJ, ya que fue determinado por la propia jurisprudencia de la Corte. Es así que vamos a observar que, en este supuesto, no queda a criterio de un tercer Estado solicitar la intervención (artículo 62) o activar el derecho que tiene para su intervención en virtud de algún tratado del que es parte (artículo 63), sino que el tercero debería participar de la controversia internacional por ser una parte indispensable para que la CIJ pueda pronunciarse sobre la misma.

La referida doctrina, también conocida como «principio del asunto del Oro Amonedado» (Zamir citado por Méndez, 2020), tiene origen en el caso Monetary Gold Removed from Rome in 1943 (Italy v. France, United Kingdom of Great Britain and Northern Ireland and United States of America) sobre el cual la CIJ manifestó que

\footnotetext{
2 Elaboración propia a partir de la información presente en la página oficial de la CIJ, los casos se encuentran ordenados cronológicamente del más antiguo al más reciente.
} 
«la resolución de la controversia afectaría jurídicamente a Albania, que no era parte en el proceso. En consecuencia, concluyó que no podría decidir sobre la controversia sin la participación de Albania» (Méndez, 2020, p. 15). El expuesto ha sido el único pronunciamiento de la CIJ respecto a este tipo de intervención, por lo que, a diferencia de los otros supuestos, no existe mayor desarrollo jurisprudencial.

$\mathrm{Al}$ respecto, nos cuestionamos el hecho de que los tribunales internacionales, por regla general (y específicamente la CIJ) no cuentan con la facultad de legislar. Al respecto, el principio Iura Novit Curia implica que el juez conozca el derecho. Sobre la base de ello, la CIJ anteriormente ha identificado la existencia de normas consuetudinarias o principios (CIJ, 1991, párrafo 188) o incluso de normas contenidas en fuentes no clásicas como los acuerdos tácitos (CIJ, 2014, párrafo 91, 102-103). Sin embargo, es de notar que las normas identificadas tienen que ser preexistentes al momento en que la CIJ las evidencia para que el principio se cumpla efectivamente, ya que, de lo contrario, se estaría dando la posibilidad a dicho tribunal de que «identifique» normas que no existen, pasando a ser más bien creadas por este.

La CIJ no cuenta con la capacidad de crear nuevas normas. Así, por ejemplo, si la $\mathrm{CIJ}$ identifica que una norma consuetudinaria no existe, como sostuvo en su opinión consultiva sobre armas nucleares (1996, párrafo 64 y ss.), no tendría la capacidad de, en su defecto, crearla. Sin embargo, el referido tipo de intervención, en donde la CIJ establece esta posibilidad de participar al ser parte indispensable, nos hace considerar que la CIJ se habría atribuido la facultad de decretar normas al haber establecido un supuesto de intervención de terceros Estados no regulado a través de su propia jurisprudencia.

De igual forma, consideramos importante señalar la posibilidad que existió de que dicho tipo de intervención se suscite en el caso Islas Marshall c. Reino Unido (2016), ya que, en dicho proceso, el Estado demandante señaló que diversos Estados nucleares habían incumplido con lo establecido en el artículo 6 del Tratado de No Proliferación de Armas Nucleares de 1968. De hecho, en razón de lo señalado es que, el Reino Unido, en su quinta excepción, manifestó que la CIJ no tendría competencia de pronunciarse sobre la controversia porque, para ello, sería necesaria la intervención de otros Estados al ser partes indispensables del proceso para un efectivo pronunciamiento. Sin embargo, cabe señalar que no fue necesario que el tribunal se pronuncie por dicha excepción preliminar, ya que admitió la primera respecto a la falta de competencia de la CIJ, conforme al artículo 36, párrafo 2 (CIJ, 2016, párrafo 58); por tanto, se declaró incompetente para manifestarse sobre la controversia internacional. 
En ese sentido, con relación a este supuesto, la CIJ ha sido mucho más renuente de volver a pronunciarse o establecer criterios específicos para su aplicación. Consideramos que es muy probable que esto se deba a los mismos motivos que la llevan a tener dicha postura hacia los otros supuestos de intervención, es decir, evitar incorporar a terceros Estados en los distintos procesos presentados.

\section{Los procedimientos para activar la intervención de terceros Estados en procesos ante la Corte Internacional de Justicia}

De manera general, las solicitudes de intervención tienen carácter incidental, lo cual significa que «no puede[n] modificar el caso principal» (Solimano, 2013, p. 45). Respecto a la intervención de un tercer Estado conforme al supuesto del artículo 62, será necesario que la petición sea depositada antes del cierre del procedimiento escrito, según lo establecido en el artículo 81 del Reglamento de la CIJ. La misma deberá consignar el nombre del agente, así como estipular el supuesto interés de orden jurídico que podría verse afectado, el objeto preciso de la intervención y todo tipo de competencia que existiese entre el solicitante y las partes en la controversia.

En cuanto al referido supuesto de intervención, consideramos pertinente señalar que la carga de la prueba recae exclusivamente en el tercero solicitante, y no se satisface con cualquier prueba, por más que el artículo $62^{3}$ contenga el término "considere». Sobre esto último, daría la impresión de que quedaría a discreción del Estado solicitante, pero ello no es correcto, toda vez que, si quedara a decisión del tercer Estado elegir qué pruebas son necesarias para su intervención, la CIJ aceptaría cualquier solicitud prevaleciendo la consideración particular de aquel.

Sobre la intervención de tercer Estado conforme al artículo 63 del Estatuto de la CIJ, el artículo 82 del Reglamento estipula que dicho Estado deberá depositar una declaración en la cual señale que desea prevalerse de su derecho de intervención. Asimismo, a diferencia de lo que sucede con el artículo 62, en este caso, tendrá que ser depositado antes de la fecha fijada para la etapa oral conforme a las disposiciones de las fuentes antes mencionadas, si bien la CIJ podría eventualmente hacer excepciones como se señalará posteriormente.

Dicho documento deberá consignar el nombre del agente, precisar el asunto y el tratado al que se refiere, así como los datos en que se basa para afirmar ser parte de la

\footnotetext{
3 Estatuto de la Corte Internacional de Justicia, artículo 62.-

«1. Si un Estado considerare que tiene un interés de orden jurídico que puede ser afectado por la decisión del litigio, podrá pedir a la Corte que le permita intervenir.

2. La Corte decidirá con respecto a dicha petición.»
} 
convención, las disposiciones del tratado cuya interpretación vendría a estar sujeta a controversia, y deberá señalar la interpretación que dicho Estado considera correcta y acompańar lo mencionado con documentos que sustenten sus posturas, según lo establecido en el artículo 82 del Reglamento.

Cabe precisar que es posible que la CIJ no proceda a notificar a los terceros que sean partes del tratado a ser interpretado conforme a lo establecido en el artículo 63 del Estatuto ${ }^{4}$. Ante ello, existe la posibilidad de solicitar la intervención, siempre que el tercer Estado "considere» que es parte del tratado cuya interpretación esté en discusión, según lo establecido en el artículo 82 del Reglamento. Al respecto, tenemos la postura de que no debería interpretarse el término "considere» en su sentido corriente, sino que el tercer Estado debería ofrecer pruebas que demuestren fehacientemente que es parte del tratado que se va a interpretar, para así evitar quizás una demora procesal innecesaria ante la CIJ.

Ante dichos supuestos, la CIJ actuará lo más inmediatamente posible, ya que le dará carácter prioritario a la solicitud. Sin embargo, de existir alguna objeción presentada por las partes, dicho tribunal tomará la decisión después de haber otorgado la oportunidad de expresarse, tanto al solicitante como a las partes (Bonafé, 2018, p. 100), conforme a lo señalado en el artículo 84 del Estatuto. De aceptarse las solicitudes, la CIJ proporcionará copias de los alegatos escritos, así como los demás anexos pertinentes, a fin de que el solicitante pueda emitir alguna declaración dentro del plazo que establezca la Corte, el cual también puede ser señalado por el presidente cuando el resto de los miembros del tribunal no estuviesen reunidos.

Asimismo, en relación con ambos supuestos de intervención, como se adelantó en la revisión del supuesto del artículo 62 del Estatuto, existe la posibilidad de que se susciten circunstancias excepcionales a los solicitantes para intervenir en el proceso elevado ante el tribunal, lo que llevará a que la CIJ valore dicha situación y analice la aceptación o no de las declaraciones de manera posterior a las etapas establecidas en las disposiciones ya mencionadas en los párrafos anteriores.

Finalmente, sobre el último supuesto existente de intervención, es decir, por ser parte indispensable, en el caso Monetary Gold Removed from Rome in 1943 (Italy v. France, United Kingdom of Great Britain and Northern Ireland and United States of America), la $\mathrm{CIJ}$ no ha establecido algún criterio en específico sobre el aspecto procesal. Sin

\footnotetext{
4 Estatuto de la Corte Internacional de Justicia, artículo 63.-

«1. Cuando se trate de la interpretación de una convención en la cual sean partes otros Estados además de las partes en litigio, el Secretario notificará inmediatamente a todos los Estados interesados.

2. Todo Estado así notificado tendrá derecho a intervenir en el proceso; pero si ejerce ese derecho, la interpretación contenida en el fallo será igualmente obligatoria para él.»
} 
embargo, nos queda rescatar que la solicitud la realizó la propia CIJ al tercer Estado a fin de consultarle si aceptaría intervenir. De aceptar, la CIJ puede declararse competente y pronunciarse sobre la controversia internacional, pero, de no aceptar, como sucedió con Albania en el caso señalado, la CIJ no contaría con un elemento indispensable para pronunciarse (CIJ, 1954, p. 34).

\section{Intervención de terceros Estados en casos ante la Corte Internacional de Justicia}

Como ya se ha mencionado, la CIJ se ha mostrado renuente a las peticiones de terceros Estados de intervenir. Sin embargo, tanto de la denegación como de la admisión de las múltiples solicitudes, se han podido desarrollar de manera más amplia los criterios de los supuestos de intervención. De igual forma, estos aún no son completamente claros.

A fin de poder brindar un mayor entendimiento respecto a los criterios esgrimidos para efectivizar los supuestos de intervención, procederemos a brindar breves ejemplos de intervención de terceros Estados en los cuales se llegó a efectivizar y en los cuales no, así como supuestos en los que, si bien no se realizó el requerimiento, se esgrimió la posibilidad de realizar la petición de intervenir en la controversia ante la CIJ.

\subsection{Casos en los que se efectivizó la intervención de terceros Estados}

La primera solicitud de intervención de un tercer Estado y que fue admitida por la CIJ fue realizada por Cuba el 15 de febrero de 1951. El referido Estado realizó la petición ante el caso Haya de la Torre (Colombia v. Perú), el cual inició el 13 de diciembre de 1950 y culminó el 13 de junio de 1951. Cabe precisar que el referido caso se puede entender como secuela del caso Asylum (Colombia v. Perú) iniciado en 1949, así como del requerimiento de interpretación del fallo del referido caso de fecha 20 de noviembre de 1950, el cual no fue admitido por la CIJ. La controversia entre el Estado peruano y Colombia tuvo como motivo el otorgamiento del asilo diplomático a favor del líder político Víctor Raúl Haya de la Torre, quien era peruano y fue perseguido por las autoridades nacionales de su Estado. Ante ello, la CIJ señaló, en un segundo proceso, que el gobierno colombiano no se encontraría obligado a entregar al político al Estado peruano.

$\mathrm{Al}$ margen de los aspectos de fondo de los casos antes mencionados, atendiendo al estudio de la intervención de terceros Estados, tendremos a Cuba como el pionero en requerir su intervención conforme al artículo 63 de la CIJ, a través de una declaración de fecha 15 de febrero de 1951 que fuese trasmitida al tribunal el 13 de marzo 
de 1951, mediante la cual manifestó ser parte de la Convención de La Habana y poseer una interpretación en específico sobre la cuestión de si Colombia se encontraría en la obligación o no de entregar al refugiado a las autoridades del Perú.

La CIJ analizó si las cuestiones señaladas en la declaración de Cuba ya habían sido discutidas en el fallo del 20 de noviembre de 1950, ya que, de ser así, no tendría sentido pronunciarse, puesto que vendría a ser cosa juzgada. En ese sentido, dicho tribunal se pronunció señalando que, efectivamente, la mayoría del contenido de la declaración ya había sido tratada y decidida en el referido fallo. Sin embargo, el agente del Gobierno de Cuba, en la audiencia pública del 15 de mayo de 1951, afirmó que existía un aspecto sobre el que no se había pronunciado la CIJ, es decir, sobre la obligación o no de Colombia de entregar al refugiado. Esto habría terminado por convencer a la CIJ, ya que admitió la intervención de Cuba el día 16 de mayo, conforme lo señala en su sentencia de fondo (CIJ, 1951b, p. 77).

$\mathrm{Al}$ respecto, consideramos que efectivamente había un interés en cuanto a la interpretación de la Convención de La Habana, ya que era el tratado esencial que estaba de por medio para la emisión de la sentencia de la CIJ. Sin embargo, creemos que no se suscitó de una manera ordenada, lo cual podría responder a que fue la primera solicitud de intervención, así como la primera en ser admitida. El motivo de lo señalado se debe a que, en los registros proporcionados por la página de la CIJ, no encontramos alguna providencia o decisión en específico en la que se hayan desarrollado los puntos de análisis de la solicitud realizada por el tribunal. Si bien es cierto que, en la sentencia de fondo se menciona la decisión de admitir la intervención de un documento del 16 de mayo, al momento de remontarnos a dicho instrumento, vamos a observar que no existe mayor desarrollo que el manifestar en un comunicado no oficial que, al haber admitido la solicitud, se procederá con la fase oral (CIJ, 1951a, $\left.\mathrm{N}^{\circ} 51 / 17\right)$.

Caso contrario observamos en la segunda solicitud presentada por Nicaragua respecto a su intervención que fuere admitida por la CIJ, conforme al artículo 62, ante el caso denominado Land, Island and Maritime Frontier Dispute (El Salvador) Honduras: Nicaragua intervening). En dicho asunto, la CIJ, quizás por la experiencia previa, emitió una «sentencia» específica de fecha 13 de setiembre de 1990, en donde detalló todos sus argumentos y emitió un pronunciamiento más ordenado, en el cual estipula que Nicaragua habría demostrado un interés legal, el cual podría ser afectado por el fallo de la CIJ (CIJ, 1990, párrafo 105).

En el caso referido existió propiamente una sentencia, pero dicha denominación varió en la siguiente solicitud de Guinea Ecuatorial, en virtud del artículo 62 del Estatuto, que fuera admitida por la CIJ, ante el caso Land and Maritime Boundary 
between Cameroon and Nigeria (Cameroon v. Nigeria: Equatorial Guinea intervening). En este supuesto, la CIJ no emitió una sentencia, sino una providencia o, por su nombre originario en inglés, order of. $\mathrm{Al}$ respecto, consideramos que ambos instrumentos tienen distinta esencia, toda vez que, como mencionan Torres Bernárdez y Moïse Mbengue «orders have to be distinguished from judgments or advisory opinions in that they contain no substantive decision» (2019, p. 986), por lo que, consideramos que la CIJ debería establecer una misma denominación de los instrumentos que recogen sus decisiones sobre las solicitudes de intervención.

Por lo expuesto, vamos a observar que no existe una uniformidad por parte de la CIJ en cuanto a cuál será el tipo de instrumento que recoja la decisión de admitir o no la solicitud realizada por el tercer Estado. Esto quizás puede generar una confusión y complicación para el estudio de los casos elevados a la CIJ, ya que resulta un poco dificultoso al momento de ubicar el instrumento preciso en el que se pronuncia la CIJ ante el referido requerimiento. Más aún cuando, de manera general, consideramos a las sentencias de la CIJ como el instrumento propio para su pronunciamiento sobre excepciones preliminares o el fondo de la controversia internacional. Por ello, creemos pertinente establecer la necesidad de la utilización de un solo término para la decisión de la CIJ en cuanto a las solicitudes de intervención, ya que hemos podido apreciar la falta de uniformidad, conforme a los cuadros detallados anteriormente, la cual, además, no responde a una continuidad temporal, ya que varían indistintamente sin importar la época.

Por otro lado, respecto al supuesto de intervención que inicialmente hemos estudiado, es decir, al realizado por Cuba, conforme al artículo 63, observamos que efectivamente se hizo el depósito requerido de la interpretación de la Convención de La Habana, en concordancia con el artículo 82 del Reglamento de la CIJ, el mismo que se suscitó antes de la etapa oral, conforme lo estableció el comunicado no oficial $\mathrm{N}^{\circ}$ 51/17 del 16 de mayo de 1951, el cual fue suscrito en la sentencia de fondo del 13 de junio de 1951.

Por último, consideramos importante señalar que, si bien se estipula como regla general el que la CIJ realice la invitación a participar a los terceros Estados que pudiesen tener interés por ser parte de un tratado relevante para la resolución del caso, conforme a lo señalado en el artículo 63 del Estatuto, en realidad, tal cual como sucedió en el caso analizado, esto puede requerirse sin haber sido notificado el Estado parte del tratado ajeno a la controversia, lo cual procederá a ser evaluado por la CIJ, en concordancia con el artículo 82 del Reglamento de la CIJ. 


\subsection{Casos en los que no se efectivizó la intervención de terceros Estados}

Por mucho tiempo se ha discutido si la CIJ tiene la discrecionalidad general de rechazar o no las solicitudes de intervención. Sin embargo, al menos en cuanto a la teoría, el propio tribunal estableció que el párrafo 2 del artículo 62 de su Estatuto no le confiere dicha competencia a raíz de la solicitud de Malta para intervenir en el caso Continental Shelf (Tunisia/Libyan Arab Jamahiriya) de 1981. Es así que, en dicho caso, vamos a observar que la CIJ "enphasizes that it does not consider paragraph 2 to confer upon it any general discretion to accept or reject a request for permission to intervene for reasons simply policy» (Rosenne, 1993, p. 33).

Efectivamente, la CIJ no posee la discrecionalidad general de decidir a su libre elección o interpretación qué solicitudes de intervención serían procedentes y cuáles no, ya que se entenderá que deberá actuar bajo los parámetros legales estipulados tanto en su Estatuto como en su Reglamento. Sin embargo, esta teoría no significa que la realidad sea concebida de la misma manera, ya que, como comenta Rossene, "the difficulty is that the law on the central question of what those limits are is far from clear or established» (1993, p. 33). Es en ese sentido que vamos a apreciar que la CIJ va a ir estableciendo los límites a la intervención en el desarrollo de su jurisprudencia, lo cual, en cierta forma, hace que sí se evidencie cierta discrecionalidad de su parte.

Respecto a esta discrecionalidad de la CIJ, se puede apreciar en la decisión de fecha 4 de mayo de 2011, en la que denegó la solicitud de intervenir del Estado de Costa Rica, quien había solicitado intervenir en virtud del artículo 62 del Estatuto, ante el caso Territorial and Maritime Dispute (Nicaragua v. Colombia), toda vez que la CIJ consideró que «no demostró que su interés de orden jurídico en el área marítima en disputa no pudiera ser protegido por el efecto relativo de las decisiones de la CIJ, de acuerdo con el artículo 59 del Estatuto de la Corte» (Sarmiento, 2012, p. 124). Esto abriría una discusión que se pensaba que ya estaba cerrada, ya que el artículo 59 menciona que la decisión de la CIJ no es obligatoria sino para las partes en litigio. Sin embargo, dicha disposición se debe interpretar en su contexto, el cual incluye a las disposiciones de intervención, concluyendo que la intervención sería un supuesto especial en el cual el tercero sí sería vinculado por la decisión de la Corte.

$\mathrm{Al}$ respecto, observamos que la falta de uniformidad en el pronunciamiento de la CIJ ante dichos requerimientos respondería, en gran medida, a los intereses particulares o decisiones de los Estados partes en el litigio, ya que, las solicitudes de Malta en el caso Continental Shelf (TunisialLibyan Arab Jamahiriya), de Italia en el caso Continental Shelf (Libyan Arab Jamahiriya/Malta), y de Costa Rica en el caso Territorial and Maritime Dispute (Nicaragua v. Colombia) no fueron admitidas por la CIJ. El común denominador en dichos casos es el que, en todos, existieron oposiciones por al menos 
una de las partes en litigio, por lo que, al parecer, la CIJ se habría dejado influenciar por las objeciones los Estados en controversia, a pesar de que «la Corte ha declarado que el consentimiento de las partes es importante pero no definitivo [...]» (Solimano, 2013, p.167).

De manera contraria, vamos a observar que la CIJ sí ha aceptado las solicitudes de intervención en casos en los que las partes de la controversia manifestaron su conformidad en cuanto a la participación de un tercer Estado. El ejemplo más claro de ello vendría a estar reflejado en la intervención de Guinea Ecuatorial ante el caso Land and Maritime Boundary between Cameroon and Nigeria (Cameroon v. Nigeria: Equatorial Guinea intervening), ya que, en este caso, tanto Nigeria como Camerún se encontraban totalmente de acuerdo con que el estado solicitante intervenga conforme el artículo 62 del Estatuto. Asimismo, al parecer, el tribunal «aceptó las solicitudes mediante órdenes bastante sucintas que no analizaban en profundidad el cumplimiento por parte de los terceros de los requisitos establecidos en las normas relevantes» (Solimano, 2013, p. 167). Asimismo, como ya señalamos, ocurrió lo propio en el caso Jurisdictional Immunities of the State (Germany v. Italy: Greece intervening), en el que Grecia ni siquiera tenía un interés jurídico tan concreto.

Conforme a lo expuesto, vamos a observar que la CIJ, solo en un caso en el que las partes no han estado de acuerdo, ha decido aceptar el requerimiento de intervención de un tercer Estado. El caso que refleja lo señalado es el de la intervención de Nicaragua ante el caso de Land, Island and Maritime Frontier Dispute (El Salvador/ Honduras: Nicaragua intervening), a pesar de la oposición de El Salvador. Aquí, la petición de Nicaragua se dio en virtud de que deseaba «informar a la Corte sobre la naturaleza de los derechos que están en cuestión en la disputa» (Sarmiento, 2012, p. 133), a lo que la Sala de la CIJ consideró como oportuno y acorde a los criterios de intervención, «teniendo en cuenta que al informar a la Corte un interviniente está asegurando que su interés de orden jurídico no será afectado» (Sarmiento, 2012, p. 133).

No obstante, nos preguntábamos a qué podría responder el hecho de que la CIJ haya normalmente denegado las solicitudes de intervención de terceros Estados en concordancia, al parecer, con la manifestación en contra de los Estados parte de la controversia en litigio respecto a la posible intervención. En ese sentido, Solimano señala que la motivación detrás de las decisiones de dicho modus operandi respondería a que la CIJ es consciente de que su funcionamiento depende de los Estados, por lo que ir en contra de las voluntades de aquellos la podría hacer susceptible de ser poco atractiva en cuanto a la elección de dicho ente como medio de solución de controversias (2013, p. 169). En ese sentido, como una hipótesis de por qué a Nicaragua sí le aceptó la solicitud de intervención pese a la negativa de las partes en 
litigio, podemos asumir que sería por el mismo motivo, pero a la inversa, teniendo en consideración que Nicaragua es el Estado con más casos en la Corte.

Por otro lado, en la solicitud de intervención de Costa Rica podemos apreciar que la CIJ decidió denegar el requerimiento, ya que el Estado le adelantó su posición, al manifestarle que oficiosamente su fallo no lo perjudicará, para lo que previamente se le solicitó que demuestre que el fallo sí podría afectarle, lo cual puede responder quizás al principio de celeridad. Consideramos esta posibilidad toda vez que la CIJ pudo haber considerado que resultaría mucho más breve y conciso el manifestarle que no tendrá ningún perjuicio, antes que aceptar su intervención y, quizás, evitar así que se dilate el proceso más de lo estipulado.

Sin embargo, lo señalado no significa que no resulte de igual manera bastante cuestionable que la CIJ exija a un Estado en cierta forma la demostración de que el fallo le podría generar un perjuicio, ya que esto podría resultar bastante subjetivo, al tratar o intentar adelantarse a la decisión del tribunal, la cual puede variar por muchos factores. Asimismo, pareciera más evidente, por la secuencia en la forma en como se ha pronunciado la CIJ, que en cierta medida la decisión de denegar la solicitud respondería de manera concreta a la oposición de los Estados ante la posible intervención. De hecho, nos da a entender que un criterio de facto para la admisión de las referidas solicitudes de intervención es el que las partes en litigio no se opongan.

Por último, en cuanto a Costa Rica, así como en los otros casos mencionados, ha cumplido con el requerimiento de forma de emitir la declaración de intervención antes de la fase escrita, es decir, el 25 de febrero de 2010, conforme al artículo 81 de la CIJ, caso contrario a lo que sucede en el supuesto de intervención por el artículo 63, el cual requiere que sea antes de la etapa oral. En ese sentido, lo único que se le cuestionó a Costa Rica es el que no pudiera emitir argumentos suficientes para la CIJ en cuanto a la afectación que conllevaría su pronunciamiento en la sentencia de fondo.

\subsection{Casos en los que existió la probabilidad de la intervención de terceros Estados}

Para concluir el presente trabajo, ya habiendo analizado y concluido diversos criterios que son tomados en cuenta para efectos de permitir o no la participación de terceros Estados en procesos ante la CIJ, nos planteamos realizar un ejercicio en el cual analicemos las posibilidades reales de participación en dos supuestos sobre los que se discutió, en su momento, respecto a dicha posibilidad, cada uno en virtud de un artículo (62 o 63), los cuales curiosamente han involucrado a nuestro Estado.

El primero de ellos fue el caso de delimitación marítima entre el Perú y Chile, en el cual se especulaba la posible participación de Ecuador en virtud del supuesto del artículo 63. Al respecto, consideramos que Ecuador efectivamente contaba con la 
posibilidad real de participar como tercer interviniente en el caso, en tanto fueron materia de interpretación tratados en los cuales este era parte junto con las partes en la controversia, como fue el caso de la Declaración de Santiago de 1952 (CIJ, 2014, párrafo 57 y ss.).

De hecho, como resalta Fuentes Véliz, la Corte efectuó la notificación a Ecuador conforme al numeral 1 del artículo 63, siendo esto no solo una necesidad sino además una obligación por parte de esta, mientras que en Ecuador recayó más bien un derecho (2010, pp. 218-219). Como ya bien sabemos, el Perú consciente de esta posibilidad inició una serie de actos diplomáticos en aras de acordar de manera definitiva el límite marítimo con Ecuador, evitando así su intervención en el caso.

Por otro lado, encontraremos que el supuesto del artículo 62 resulta mucho más restrictivo, siendo el ejemplo de ello la discusión sobre si el Estado peruano habría podido intervenir en la controversia entre Bolivia y Chile, la misma que fuese elevada ante la CIJ el día el 24 de abril de 2013. Sobre ello, algunos especialistas en la materia manifestaron, en su momento, que no era posible que el Perú alegue intervenir en el litigio, más allá del resultado de la CIJ sobre el fondo del caso, ya que se entendería que su resultado no podía afectar en ningún sentido al Perú (Abugattas, 2015), afirmación que compartimos.

A fin de un mejor entendimiento, consideramos pertinente informar brevemente sobre el caso en mención. Bolivia decidió demandar a Chile en virtud del artículo XXXI del Pacto de Bogotá de 1948, alegando la existencia de la obligación de negociar un acceso soberano al océano Pacífico, la cual presuntamente se encontraría en distintos instrumentos presentados ante la CIJ. En ese sentido, le correspondía a la CIJ analizar si efectivamente se recogía la referida obligación en los principios generales del derecho internacional, en tratados o en otras fuentes del derecho internacional presentadas por el Estado de Bolivia.

Si bien ya sabemos que la CIJ falló en contra de Bolivia el 1 de octubre de 2018, la disyuntiva se generaba con relación a si es que fallaba a favor de dicho Estado, alegando así la existencia de la obligación de negociar un acceso soberano al mar. En ese sentido, deberemos tomar en cuenta lo seńalado por la CIJ en la sentencia de excepciones preliminares del referido caso de fecha 15 de junio de 2014, ya que en su párrafo 33 manifestó que si Bolivia quisiera alegar una obligación de resultado "Even assuming arguendo that the Court were to find the existence of such an obligation, it would not be for the Court to predetermine the outcome of any negotiation that would take pleace in consequence of that obligation» (CIJ, 2015, párrafo 33).

En ese sentido, a dicha obligación no se le hubiese podido requerir un resultado, ya que la referida obligación es «un intento genuino de emprender discusiones con la 
otra parte, con el fin de resolver una disputa» (Infante, 2019, p. 54) o también se puede traducir en «la convicción de estar actuando, en todo momento, conforme al derecho y justicia, de manera leal y oportuna en virtud de la deseable reciprocidad que ha de existir y fomentarse en las relaciones entre los diversos actores del ámbito supranacional» (Benfeld y Müller, 2018, p. 75), es decir, realizar todas las acciones necesarias a fin de poder llegar a un resultado anhelado, el mismo que no necesariamente se logra alcanzar.

Por lo expuesto, como podemos observar, no existía posibilidad de que la CIJ estableciera la obligación de alcanzar un resultado en específico, es decir, de otorgar un acceso soberano al mar en favor de Bolivia. Esto solo se hubiese podido alegar si, en un tratado, hubiesen acordado ello de manera específica o si se estuviese en el supuesto reflejado en la Opinión Consultiva de 1996 ante la CIJ, sobre la licitud de armas nucleares, ya que en dicha OC se estableció que sí había una obligación de resultado conforme a lo establecido en el artículo VI del Tratado sobre la no proliferación de armas nucleares, toda vez que el no resultado podría generar un peligro para la comunidad internacional (CIJ, 1996, párrafo 99). A esta conclusión llegó la CIJ, pero tomando en consideración el perjuicio global, supuesto en el que no encajaría lo alegado por Bolivia, como tampoco en el de la existencia de un tratado en vigor que señale la referida obligación de resultado.

En ese sentido, recordando lo establecido en el artículo 62 del Estatuto, para intervenir en una controversia internacional elevada ante la CIJ será necesario demostrar la afectación a un interés de orden jurídico. Dado ello, en el caso señalado, el Perú nunca habría podido alegar que el resultado de la CIJ le hubiese podido generar un dańo, ya que como hemos manifestado, lo máximo que pudo haber dispuesto la CIJ era la obligación de que Bolivia y Chile se pusieran a negociar, sin necesidad de alcanzar un resultado concreto. Asimismo, por más que, en un supuesto, ambos Estados hayan llegado a un acuerdo en la negociación, esto no hubiese comprometido al Estado peruano, ya que nada podría afirmar que el acceso que Chile le hubiese brindado a Bolivia haya tenido que darse por el territorio de Arica, en el que el Perú tiene potestad en virtud del Protocolo Complementario del Tratado de Lima de 1929.

No obstante, suponiendo que se hubiese tratado de una obligación de resultado (el cual, como hemos señalado, no es el caso), la CIJ habría determinado que Chile le otorgue a Bolivia un acceso soberano al mar. Sin embargo, el referido tribunal no hubiese tenido la potestad de imponer por qué parte del territorio le brindaría ese acceso, a menos que hubiese existido un tratado que así lo hubiese estipulado. De no ser el caso, la CIJ no habría podido ir en contra de la soberanía del Estado peruano imponiéndole que se le brinde el acceso a Bolivia por un territorio sobre el cual 
tendría decisión, ya que Chile y el Perú tienen la obligación en vigor de consultarse entre sí sobre cualquier decisión respecto a los territorios de Tacna y Arica.

Por lo mencionado, en ningún supuesto, hubiese cabido la intervención del Perú conforme al artículo 62 del Estatuto, ya que jurídicamente hablando era imposible que la $\mathrm{CIJ}$ se pronunciara afectando algún derecho o interés jurídico del Estado peruano. Por esos motivos consideramos que no habría sido viable que la CIJ, bajo los criterios estudiados, aceptara una eventual solicitud de intervención del Perú en el caso de Bolivia y Chile. Cabe precisar que, respecto a este caso, no hemos mencionado en adición al artículo 63 del Estatuto porque aquel se basa en la intervención en cuanto a la interpretación de un tratado, pero en el referido caso no existió tratado alguno en el que se haya cuestionado la participación del Perú.

\section{Conclusiones}

Conforme a lo estudiado, podemos apreciar que los criterios para los tipos de intervención establecidos en el Estatuto (artículos 62 y 63) han sido desarrollados por la jurisprudencia de la $\mathrm{CIJ}$, ya que los que se encuentran establecidos en dicho instrumento, así como en el reglamento de la CIJ, no brindan los lineamientos necesarios para que la CIJ pueda pronunciarse sin problema alguno. Por ello, en diversas oportunidades el referido tribunal ha tenido que interpretar las propias disposiciones establecidas.

Asimismo, hemos podido observar que los criterios establecidos por la CIJ para tomar decisiones sobre la admisión de la solicitud de los terceros Estados varían según el caso lo requiera y, más bien, han estado respondiendo, en casi su totalidad, a las posiciones de los Estados en litigio. Esto se evidencia, ya que, si estos manifiestan su inconformidad en que un tercer Estado intervenga, la CIJ, aparentemente, tiene preferencia por pronunciarse acorde al sentir de los Estados en controversia, lo cual ha llevado a que, en algunas oportunidades, no responda del todo acorde a derecho, como fue en el caso de la solicitud de intervención de Costa Rica en el caso Territorial and Maritime Dispute (Nicaragua v. Colombia).

Como ya habíamos mencionado, consideramos probable que el actuar de la CIJ responda a que no desea dejar de ser una de las mejores opciones como medio de solución de controversias internacionales a la que los Estados deseen recurrir, ya que son estos quienes le han dado y le seguirán dando las atribuciones para pronunciarse sobre las controversias que se susciten con otros Estados. Sin embargo, consideramos que, para un mayor orden y entendimiento, el referido tribunal debería pronunciarse conforme a derecho y evitar subjetividades, así como también uniformar sus pronunciamientos e instrumentos en que estos se contienen. 


\section{Referencias bibliográficas}

Abugattas, G. (2015). Entrevista sobre la sentencia de excepción preliminar emitida por la Corte Internacional de Justicia sobre el diferente entre Bolivia y Chile. Ius Inter Gentes. [Archivo de video en la plataforma Youtube] https://www.youtube.com/ watch?v=Zwkv5rKALuI\&ab_channel=IusInterGentes

Benfeld, E. y Karl, G. (2018). ¿Qué significa en el ámbito del derecho internacional público estar obligado a negociar de buena fe? Precisiones conceptuales y posición de la Corte Internacional de Justicia en esta materia, a propósito del rechazo a la objeción preliminar presentada por Chile ante dicha Corte con ocasión de la demanda boliviana de 2013. Ius Et Praxis, (24), 69-100. https://scielo.conicyt.cl/scielo. php?script=sci_abstract\&pid=S0718-00122018000100069\&lng=es\&nrm=iso

Bonafé, B. (2018). Discretional intervention (Article 62, Statute of the Court). En P. Wojcikiewicz y J. Rodrígues (Eds.), Latin America and the International Court of Justice (pp. 88-119). Routledge. https://doi.org/https://doi.org/10.4324/9781315717890

Corte Internacional de Justicia [CIJ]. (1951a). Haya de la Torre (Colombia v. Perú). Comminiqué $\mathrm{N}^{\circ}$ 51/17 (unofficial) del 16 de mayo de 1951. https://www.icj-cij.org/ public/files/case-related/14/12017.pdf

Corte Internacional de Justicia [CIJ]. (1951b). Haya de la Torre (Colombia v. Perú). Sentencia de fondo del 13 de junio de 1951. https:/www.icj-cij.org/public/files/caserelated/14/014-19510613-JUD-01-00-EN.pdf

Corte Internacional de Justicia [CIJ]. (1954). Monetary Gold Removed from Rome in 1943 (Italy v. France, United Kingdom of Great Britain and Northern Ireland and United States of America). Sentencia de fondo de 15 de junio de 1954. https://www.icj-cij. org/public/files/case-related/19/019-19540615-JUD-01-00-EN.pdf

Corte Internacional de Justicia [CIJ]. (1981a). Case Concerning the Continental Shelf (Tunisia/Libyan Arab Jamahiriya). Observaciones escritas a la solicitud de Malta para intervenir del 25 de febrero de 1981. https://www.icj-cij.org/public/files/caserelated/63/063-19810225-WRI-01-00-BI.pdf

Corte Internacional de Justicia [CIJ]. (1981b). Case Concerning the Continental Shelf (Tunisia/Libyan Arab Jamahiriya). Sentencia sobre la solicitud de Malta para intervenir del 14 de abril de 1981. https://www.icj-cij.org/public/files/case-related/63/06319810414-JUD-01-00-EN.pdf

Corte Internacional de Justicia [CIJ]. (1983). Case Concerning the Continental Shelf (Libyan Arab Jamahiriya/Malta). Observaciones escritas a la solicitud de Italia para intervenir del 5 de diciembre de 1983. https://www.icj-cij.org/public/files/case-related/68/9583. pdf

Corte Internacional de Justicia [CIJ]. (1984). Continental Shelf (Libyan Arab Jamahiriya/ Malta). Sentencia sobre la solicitud de Italia para intervenir del 21 de marzo de 1984. https://www.icj-cij.org/public/files/case-related/68/068-19840321-JUD-01-00-EN. pdf

Corte Internacional de Justicia [CIJ]. (1986). Military and Paramilitary Activities in and against Nicaragua v. United States of America. Sentencia de fondo de fecha 27 de 
junio de 1986. https:/www.icj-cij.org/public/files/case-related/70/070-19860627JUD-01-00-EN.pdf

Corte Internacional de Justicia [CIJ]. (1990). Land, Island and Maritime Frontier Dispute (El Salvador/Honduras: Nicaragua intervening). Sentencia sobre la solicitud de Nicaragua para el permiso de intervenir del 13 de setiembre de 1990. https://www. icj-cij.org/public/files/case-related/75/075-19900913-JUD-01-00-EN.pdf

Corte Internacional de Justicia [CIJ]. (1996). Advisory Opinion Legality of the Threat or Use of Nuclear Weapons. Opinión consultiva del 8 de julio de 1996. https://www.icj-cij. org/public/files/case-related/95/095-19960708-ADV-01-00-EN.pdf

Corte Internacional de Justicia [CIJ] (1999a). Land and Maritime Boundary between Cameroon and Nigeria (Cameroon v. Nigeria) Equatorial Guinea requests permission to intervene in the proceedings. Comminiqué $\mathrm{N}^{\circ}$ 99/35 (unofficial) del 30 de junio de 1999. https://www.icj-cij.org/public/files/case-related/94/094-19990630PRE-01-00-EN.pdf

Corte Internacional de Justicia [CIJ]. (1999b). Land and Maritime Boundary between Cameroon and Nigeria (Cameroon v. Nigeria). Providencia del 21 de octubre de 1999. https://www.icj-cij.org/public/files/case-related/94/094-19991021-ORD-0100-EN.pdf

Corte Internacional de Justicia [CIJ]. (2011). Jurisdictional Immunities of the State (Germany v. Italy: Greece intervening). Providencia del 4 de julio de 2011. https://www.icj-cij. org/public/files/case-related/143/143-20110704-ORD-01-00-EN.pdf

Corte Internacional de Justicia [CIJ]. (2014). Maritime Dispute (Perú v. Chile). Sentencia de fondo de 27 de enero de 2014. https://www.icj-cij.org/public/files/caserelated/137/137-20140127-JUD-01-00-EN.pdf

Corte Internacional de Justicia [CIJ]. (2015). Obligation to negotiate access to the Pacific Ocean. Sentencia sobre excepciones preliminares del 24 de setiembre de 2015. https:// www.icj-cij.org/files/case-related/153/153-20150924-JUD-01-00-EN.pdf.

Corte Internacional de Justicia [CIJ]. (2016). Obligations concerning negotiations relating to cessation of the nuclear arms race and to nuclear disarmament (Marshall Island v. United Kingdom). Sentencia sobre excepciones preliminares del 5 de octubre de 2016. https://www.icj-cij.org/files/case-related/160/160-20161005-JUD-01-00-EN. pdf

Corte Internacional de Justicia [CIJ]. (2018) Obligation to negotiate access to the Pacific Ocean. Sentencia de fondo del 1 de octubre de 2018. https:/www.icj-cij.org/files/ case-related/153/153-20181001-JUD-01-00-EN.pdf.

Fuentes Véliz, J. A. (2010). A propósito de la notificación a Ecuador, por parte de la Corte Internacional de Justicia, respecto al diferendo limítrofe marítimo entre Perú y Chile. Advocatus, (23), 217-222. https://revistas.ulima.edu.pe/index.php/Advocatus/article/ download/3137/3046/

Novak, F. y García-Corrochano, L. (2019). Derecho Internacional Público. Tomo III. Otros Sujetos de Derecho Internacional y Solución de Controversias. $2 a$ ed. Thomson Reuters. 
Infante, M. (2019). La Corte Internacional de Justicia se pronuncia sobre la demanda de Bolivia contra Chile relativa a una obligación de negociar. La sentencia de 01 de octubre de 2018. Revista Española de Derecho Internacional, (71), 49-74. https:// dialnet.unirioja.es/servlet/articulo? codigo $=7078074$

Méndez Chang, E. (2019). Las controversias internacionales en la jurisprudencia reciente de la Corte Internacional de Justicia. En F. Novak (Ed.), Cambios y transformaciones en el Derecho Internacional en el siglo XXI. Estudios en homenaje a la Facultad de Derecho PUCP en su centenario (pp. 13-37). Facultad de Derecho de la Pontificia Universidad Católica del Perú.

Negro, D. (2012). La inmunidad jurisdiccional de los Estados: el caso Alemania contra Italia - Corte Internacional de Justicia (2012). Agenda Internacional, 19(30), 243-262. https://revistas.pucp.edu.pe/index.php/agendainternacional/article/view/6269

Rosenne, S. (1993). Intervention in the International Court of Justice. Martinus Nijhoff Publishers.

Sarmiento, A. (2012). La Corte Internacional de Justicia y la intervención de terceros en cuestiones marítimas: a propósito de la decisión en las solicitudes de intervención de Costa Rica y Honduras en la Controversia territorial y marítima (Nicaragua vs. Colombia). Anuario Colombiano de Derecho Internacional, (5), 123-151. https://revistas.urosario. edu.co/index.php/acdi/article/view/2377

Solimano, L. (2013). Intervención de terceros ante la Corte Internacional de Justicia. (Tesis de licenciatura, Universidad de Chile, Facultad de derecho, Departamento de Derecho Internacional. Santiago, Chile). http://repositorio.uchile.cl/handle/2250/115317

Torres Bernárdez, S. y Moïse Mbengue, M. (2019). Article 48. En A. Zimmermann, C. Tams, K. Oellers-Frahm y C. Tomuschat (Eds.), The Statute of the International Court of Justice (pp. 984-1028). 2a Ed. Oxford University Press. https://doi: 10.1093/ law/9780198814894.001.0001

Fecha de recepción: 16 de abril de 2021 Fecha de aprobación: 17 de agosto de 2021 